Review

\title{
Relative Effectiveness of Cell-Cultured versus Egg-Based Seasonal Influenza Vaccines in Preventing Influenza-Related Outcomes in Subjects 18 Years Old or Older: A Systematic Review and Meta-Analysis
}

\author{
Joan Puig-Barberà ${ }^{1, *}$, Sonia Tamames-Gómez ${ }^{2}$, Pedro Plans-Rubio ${ }^{3}$ and José María Eiros-Bouza ${ }^{4}$ \\ 1 FISABIO, Área de Investigación en Vacunas, 46020 Valencia, Spain; jpuigb55@gmail.com \\ 2 Consejería de Sanidad, Dirección General de Salud Pública, Servicio de Información en Salud Pública, \\ 47001 Valladolid, Spain; sonia.tamames@jcyl.es \\ 3 Agència de Salut Pública de Catalunya, Departament de Salut, 08005 Barcelona, Spain; pedro.plans@gen- \\ cat.cat \\ 4 Hospital Universitario “Río Hortega”, 470129 Valladolid, Spain; eiros@med.uva.es \\ * Correspondence: jpuigb55@gmail.com; Tel.: +34-67995698
}

\begin{abstract}
Avian mutations in vaccine strains obtained from embryonated eggs could impair vaccine effectiveness. We performed a systematic review and meta-analysis of the adjusted relative vaccine effectiveness (arVE) of seed cell-cultured influenza vaccines (ccIV) compared to egg-based influenza vaccines (eIV) in preventing laboratory-confirmed influenza related outcomes (IRO) or IRO by clinical codes, in subjects 18 and over. We completed the literature search in January 2021; applied exclusion criteria, evaluated risk of bias of the evidence, and performed heterogeneity, publication bias, qualitative, quantitative and sensitivity analyses. All estimates were computed using a random approach. International Prospective Register of Systematic Reviews, CRD42021228290. We identified 12 publications that reported 26 adjusted arVE results. Five publications reported 13 laboratory confirmed arVE and seven reported 13 code-ascertained arVE. Nine publications with 22 results were at low risk of bias. Heterogeneity was explained by season. We found a significant $11 \%$ ( 8 to $14 \%$ ) adjusted arVE favoring cCIV in preventing any IRO in the 2017-2018 influenza season. The arVE was 3\% (-2\% to 7\%) in the 2018-2019 influenza season. We found moderate evidence of a significant advantage of the ccIV in preventing IRO, compared to eIV, in a well-matched $\mathrm{A}(\mathrm{H} 3 \mathrm{~N} 2)$ predominant season.
\end{abstract}

Keywords: adults; influenza; cell-cultured vaccine; egg-based vaccine; influenza vaccine; relative vaccine effectiveness; real word evidence; mutation; human/prevention \& control*; comparative study

\section{Introduction}

Influenza is a severe yearly threat to human health [1], especially in those at increased risk due to age or underlying medical conditions [2]. Influenza A and B viruses are responsible for yearly seasonal epidemics. The economic and health impact of influenza epidemics depends on virus characteristics, population immunity and preventive measures [3]. It has been reported that $\mathrm{A}(\mathrm{H} 1 \mathrm{~N} 1) \mathrm{pdm} 09$ has a severe effect on young adults [4], $\mathrm{B} /$ Victoria-lineage in children [5], B/Yamagata-lineage shows a bimodal age distribution, affecting older ages than $\mathrm{B} /$ Victoria-lineage [5], and $\mathrm{A}(\mathrm{H} 3 \mathrm{~N} 2)$ is especially severe in the elderly [6]. Each year, seasonal influenza epidemics cause worldwide an estimated 3 to 5 million severe illnesses and 290,000 to 650,000 deaths [7-9].

Vaccines are the main line of protection against influenza [10]. Still, vaccine effectiveness in preventing influenza-related illness ranges from mild to moderate, with $40 \%$ to $60 \%$ effectiveness due to virus variability [11], the mismatch between the vaccine and 
circulating strains, the primed immune response to similar previous influenza infections and the mild immunodeficiency associated to age or underlying conditions [12]. Avian mutations in vaccine strains obtained from embryonated eggs also impair vaccine performance [12-14].

In the last thirty years, in addition to the annual update of the vaccine composition to the presumed circulating strains [15], various strategies have been adopted to outdo the limitations of the traditional seasonal influenza vaccines, such as enhancing immunogenicity with new administration routes, mucosal or intradermal [16], the addition of adjuvants [17] or increasing the amount of antigen in the vaccine [18,19] and improving the vaccine strain match to circulating viruses by obtaining the vaccine antigen by recombination [20] or by culture in mammalian cells [21] to elude avian adaptative mutations along the vaccine manufacturing process [22].

Fully cell-cultured influenza vaccines (ccIV), from strain selection to manufacturing, were first licensed for their use in humans in the United States (U.S.) in 2016 [23]. For the influenza vaccines available before the 2019-2020 season, only the A(H3N2) component was obtained by cell-culture, while the $\mathrm{A}(\mathrm{H} 1 \mathrm{~N} 1) \mathrm{pdm} 09$ and $\mathrm{B}$ vaccine components were obtained by egg-culture [24]. Cell-derived B lineages were added in the vaccines distributed in the 2018-2019 [25] and, since the 2019-2020 season, all the strains in the cell-culture vaccine used in the U.S. were obtained by cell-culture. The four strains cell-derived quadrivalent influenza vaccine was licensed in Europe for the 2017-2018 season [26].

A recent systematic review on the effectiveness of newer seasonal influenza vaccines [27] concluded that while it is assumed that ccIV may be more effective than traditional egg-based vaccines (eIV) due to reduced antigenic mutation during vaccine production, there are limited data to assess the effectiveness of ccIV compared with eIV and that the evidence regarding the comparability of newer vaccines to traditional seasonal influenza vaccines is uncertain due to a lack of available literature.

Various new recent publications, not included in the mentioned review, reported the adjusted relative vaccine effectiveness (arVE) of ccIV compared to eIV [28-35]. We performed a systematic review of the recently published evidence on relative vaccine effectiveness of ccIV compared to eIV in preventing influenza-related outcomes (IROs).

\section{Materials and Methods}

We followed the Preferred Reporting Items for Systematic Reviews and Meta-Analyses (PRISMA) guidelines for the protocol, conduct and reporting [36-38]. We registered the protocol in the International Prospective Register of Systematic Reviews (PROSPERO), CRD42021228290.

Our objective was to perform a systematic literature review of the existing evidence on the arVE of ccIV, compared to eIV in preventing $\mathrm{A}(\mathrm{H} 3 \mathrm{~N} 2)$ related IROs in subjects 18 years old or older. As secondary objectives, we looked to evaluate the arVE of ccIV, compared to eIV in preventing IRO with influenza, and IRO related either to A(H1N1)pdm09, $\mathrm{B}$ overall or $\mathrm{B} /$ lineage or IRO determined by specific clinical codes in subjects 18 years old or older. We defined IRO as any clinical outcome related to influenza, determined as IRO with laboratory-confirmed influenza or IRO with influenza-specific clinical codes.

We performed our search in PubMed, using the terms Influenza AND Vaccin* AND (Effectiv* OR Effic ${ }^{*}$ ) AND (relativ* OR compara*) AND cell, and subsequently adapted the search strategy to the Web of Science (WoS) All Databases and Core Collection, medRxiv and bioRxiv. In all cases, we performed the search since the inception of the databases and without restrictions in publication type or language. We aimed to saturation by checking the reference lists of relevant publications, scientific meetings, guidelines, reviews and the authors' archives. Finally, we contacted the authors of retrieved publications for additional data, results or clarification. We repeated the same search strategy after data extraction, but before performing the data analysis.

\subsection{Study Selection}


We downloaded the search results into the Rayyan platform [39]. After trimming duplicates, two authors screened the titles and abstracts for inclusion. We excluded animal studies, case studies, immunogenicity studies, studies on pandemic or pre-pandemic vaccines, zoonotic vaccines, and considered for inclusion non-duplicated randomised clinical trials and non-randomised studies of the effect of interventions in humans, 18 years old and over; that reported arVE results in preventing IRO comparing ccIV with eIV. Each author judged independently and blinded to the other author's selection the compliance with the inclusion criteria of the retrieved publications. After finishing this first selection, we unblinded the Rayyan platform. The four authors agreed on the publications for inclusion.

\subsection{Data Extraction}

From each publication that we considered for inclusion, we obtained the full text. We searched again in the full text according to criteria for duplicates, defined as publications with the same authors, season, population, age-groups and analysis. When we found duplicates, we chose papers in preference of conference proceedings or abstracts.

We extracted from the non-duplicated publications full text and supplementary documents the second name of the first author, journal or conference name, volume, first page or abstract number, publication year, season of reported results, geographical location, population source, age groups, study setting, study design; statistical method, measured outcomes, the method used to determine the outcomes (laboratory or clinical codes), control of confounders such as sex, race, underlying conditions, frailty, previous health care use, antivirals, days from symptoms onset to specimen collection, vaccination date, calendar time, previous vaccination, the method used to find out the vaccines administered, number of IRO and vaccinated subjects by vaccine type, arVE results, arVE confidence intervals (CI), and funding source. We checked again for compliance with inclusion criteria, excluded those results that did not comply with our inclusion criteria, and recorded the reasons for exclusion.

\subsection{Study Quality Assessment}

The four authors evaluated the quality of the publications independently by assessing the risk of bias (RoB) following the ROBINS-I guidelines and answering signaling questions using a modified ROBINS-I template [40].

We considered bias due to confounding, selection bias, classification bias, comparability and exchangeability, attrition bias, bias in the measurement of the outcomes, and outcome reporting bias. Each overall publication RoB was judged as low (the study was comparable to a well-performed randomised trial), moderate (the study was sound for a non-randomised study but could not be considered close to a well-performed randomised trial), serious (the study had some important problems) or critical (the study was too problematic to provide any valid evidence on the effects of the intervention). We resolved differences by consensus. We generated the plots showing the RoB by publication and domain using the ROBVIs tool[40]. We excluded publications with serious or critical RoB bias.

\subsection{Data Analysis}

We performed a descriptive analysis according to season, age group, study design, outcome setting (primary care, hospital or both), outcome determination method, funding and RoB.

Depending on the study design, the published results and their confidence intervals were incidence rates ratios, risk ratios or adjusted odds ratios. Under the rare-diseases assumption, we assumed these measures as unbiased estimators of the adjusted relative risk (aRR) [41], and arVE as $(1-\mathrm{aRR}) \times 100$ [42], for the metanalysis calculations we entered the log of the aRR obtained from 1-(arVE/100), and the standard errors as (ln aRR upper limit - ln aRR lower limit) / 3.92 . 
When we identified mutually-dependent results, and to avoid repeated contribution bias, we used the more encompassing and relevant arVE IRO result and report, in each case, the included and excluded results. We defined mutually-dependent results when a publication reported more than one arVE in preventing diverse defined IROS for the same age-group and season.

We evaluated the existence of outliers and heterogeneity among the included publications' results by Galbraith and forest plots. After excluding mutually-dependent results, we considered the hypothesis of no difference in results between subgroups when the bilateral $p$-value for the Cochran's Q statistic was $>0.10$ [43] and the presence of heterogeneity when $\mathrm{I}^{2}$ was $\geq 50 \%$ [44]. We explored the degree to which heterogeneity of the results was influenced by season, age, study setting, study design, IRO determination method, funding and RoB.

We explored publication bias and asymmetry of reported results with funnel plots and the test of Egger [45], considering all reported included results.

We performed a quantitative meta-analysis and computed the aggregate aRR, and aRR 95\%CI when the following quality and homogeneity criteria were satisfied: low or moderate RoB, Q $p$-value $>0.10 ; \mathrm{I}^{2}<50 \%$, no evidence of publication bias, and three or more results available after excluding mutually-dependent results.

We performed a sensitivity analysis of the quantitative meta-analysis by non-parametric trim and fill estimation of not included results and the imputed pooled result and repeated the same calculations without excluding mutually-dependent results [46]. We estimated the impact of not including one result, result by result, in the estimated pooled aRR and aRR 95\%CI. Finally, we evaluated the effect of any outlier results on the overall results and their heterogeneity.

We performed all calculations under a conservative random-effects approach, assuming clinical and methodological heterogeneity between studies by restricted maximum likelihood [47] using STATA v. 17: StataCorp LLC. College Station, TX, USA.

\section{Results}

We performed the bibliographic search between the 7th and 12th of January 2021 and retrieved 5393 publications. We identified nine additional publications [48-52] in two recent influenza conferences (Options X 2019 and ESWI 2020) and the author's datasets [30,53-55], adding to 5402 retrieved publications. Two authors screened the titles and abstracts, and the four authors reviewed and agreed on the screening result. We identified 18 publications [28,30-32,48-57] for full-text review (Figure 1). We performed a new search on the 1 April 2021 and did not identify additional publications. 
5,393 publications recovered: 592 PubMed

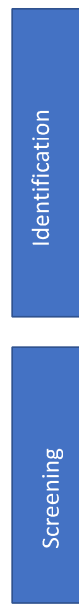

1,502 Web of Science all

482,Web of Science Core

707 medRxiv

2110 bioRxiv

9 publications from

other sources
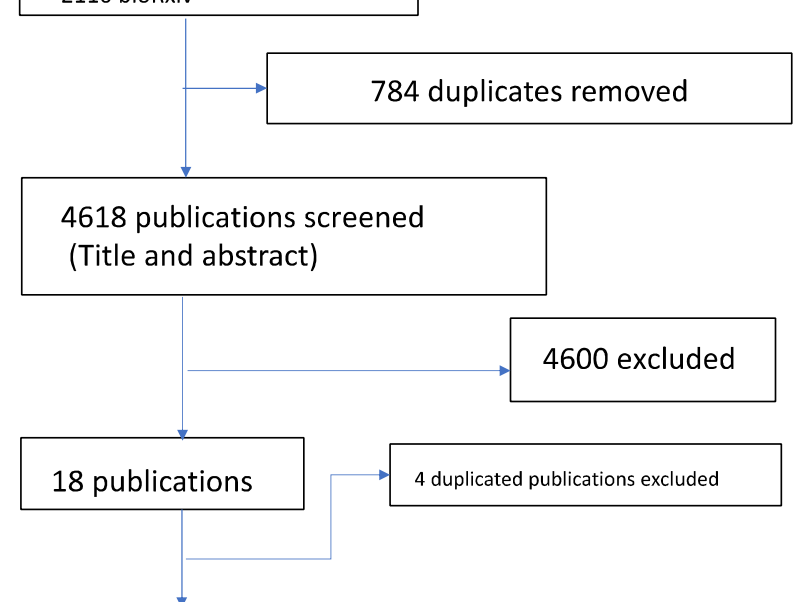

.
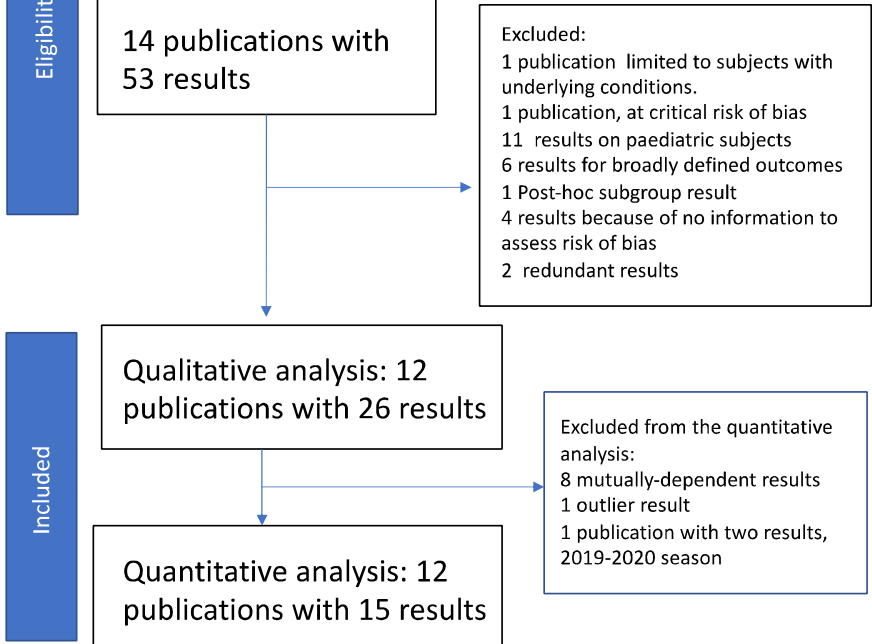

Qualitative analysis: 12 publications with 26 results

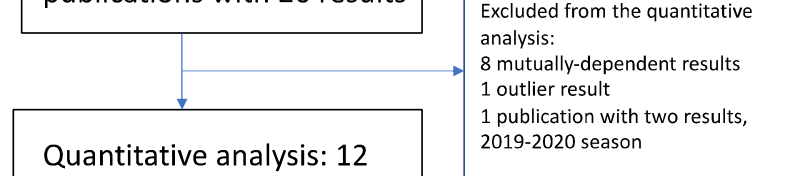

publications with 15 results

Figure 1. PRISMA flow diagram. See main text and Table S1 for details. PRISMA: Preferred Reporting Items for Systematic reviews and Meta-Analyses.

Among the 18 publications, we excluded four conference abstracts $[30,48,49,51]$ with duplicated results published in three journal papers [53,29,57]. The remaining 14 publications [28,29,31-35,50,52-57] reported 53 results (Figure1, Table S1). We checked compliance with reporting IRO in subjects $\geq 18$ years. That process resulted in the exclusion of one publication [50] and a total of 18 results related to broadly defined outcomes or results on populations including paediatric age groups (Table S1). We assessed the risk of bias in the remaining 13 publications, resulting in excluding one publication [31] and a total of nine results (Table S1). Overall, the leading contributors to bias in the retrieved studies were the lack of information on the distribution of missing data, measurement of outcomes and selection of participants (Figure 2). 

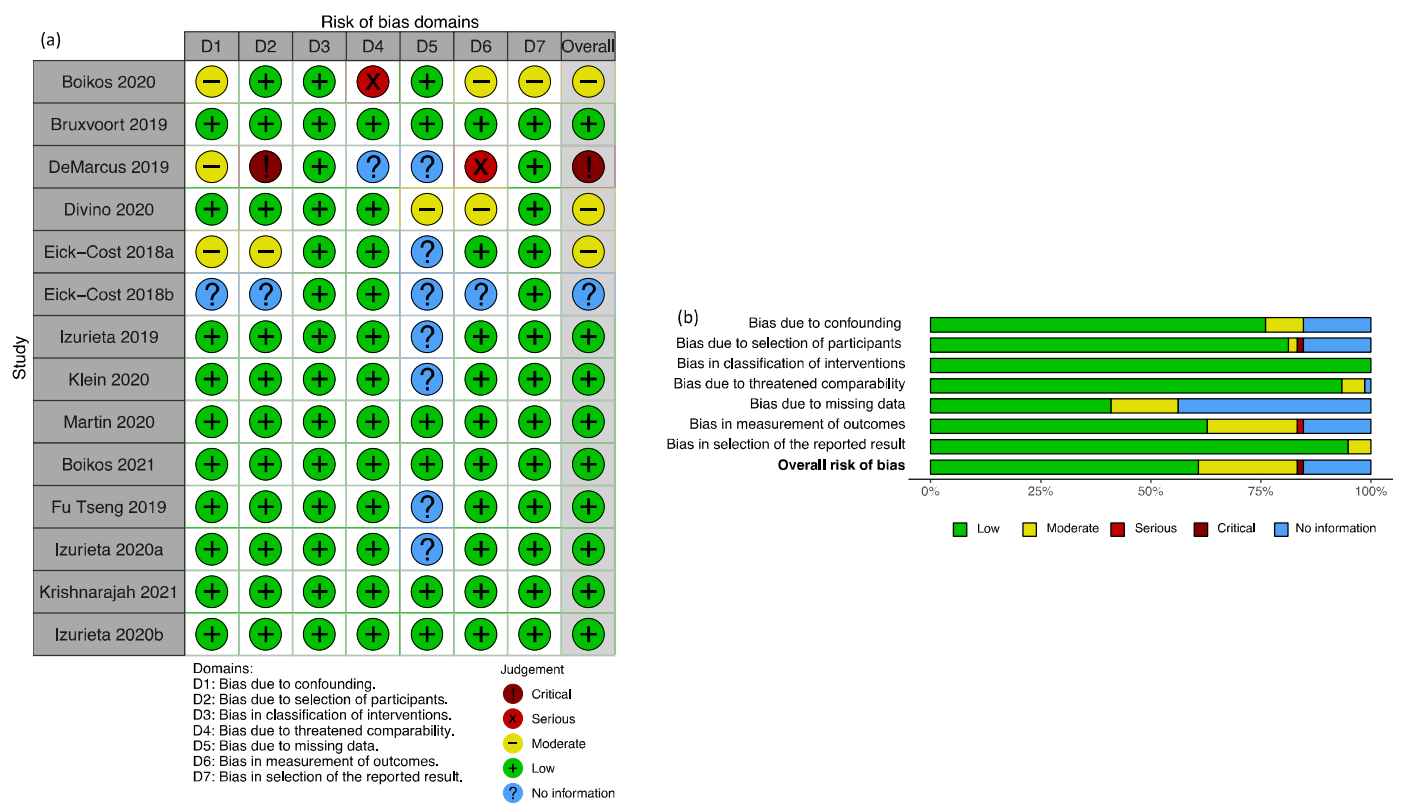

Figure 2. Risk of bias evaluation summary: (a) Judgement about risk of bias by domain in each recovered publication reporting results on the relative effectiveness of cell-cultured compared to egg-based vaccines. (b) Weighted contribution of each domain to the assessed risk of bias in the included publications.

\subsection{Qualitative Review of Included Publications}

We included in our qualitative review 12 publications [28,29,32-35,52-57] that reported 26 results (Figure 1). We describe the main characteristics of the publications and the reported results in Tables 1 and 2. All the included publications were conducted in the U.S. Seven publications reported 14 results in the 2017-2018 influenza season $[28,29,32,34,35,54,55]$, four publications reported ten results in the 2018-2019 season $[33,52,53,57]$, and one publication reported two results in the 2019-2020 season [56]. Eight publications reported 15 retrospective-cohort results [29,32-35,56,57], and four publications reported 11 test-negative results $[28,52,54,55]$. One publication reported three results in the 18 and over age-group [55]. Six publications reported seven results in the 18 to 64 age group $[29,34,35,53,54,57]$, and seven publications reported 16 results in the $\geq 65$ years of age-group $[28,32,33,35,52,56,57]$. Two publications reported three results in preventing primary care IRO [32,35], eight publications reported 18 results in preventing hospital IRO $[28,29,32,33,52,53,55,56]$, and three publications reported five results in preventing IRO defined as medical encounters (encompassing outpatient and inpatient IROs) [34,54,57]. Five publications (one retrospective cohort and four test-negative) reported 13 results based on laboratory-confirmed IRO $[28,34,52,54,55]$, and seven reported 13 results determined by clinical codes $[32-35,53,56,57]$. Five publications with eight results were pharma funded $[28,29,35,53,57]$, whereas seven with 18 results were non-pharma funded [32$34,52,54-56]$. Finally, according to RoB, nine at low RoB publications reported 22 results $[28,32-34,52,53,55-57]$, and three at moderate risk of bias reported four results $[29,35,58]$. 
Table 1. Studies included in the qualitative systematic review of adjusted relative vaccine effectiveness of cell versus egg-derived influenza vaccines in subjects 18 years old or older.

\begin{tabular}{|c|c|c|c|c|c|c|c|c|c|c|c|c|c|c|c|}
\hline Author, Year & Season & $\begin{array}{l}\text { Geographic } \\
\text { Location }\end{array}$ & Study Design & $\begin{array}{l}\text { Age } \\
\text { Group }\end{array}$ & Outcome * & $\begin{array}{l}\text { Outcome } \\
\text { Determination } \\
\text { Method }^{+}\end{array}$ & $\begin{array}{c}\text { Risk } \\
\text { of } \\
\text { Bias }\end{array}$ & $\begin{array}{c}\text { Cell- } \\
\text { Cultured } \\
\text { IV } \\
\text { (n) }\end{array}$ & $\begin{array}{c}\text { Influenza } \\
\text { Related } \\
\text { Outcomes } \\
\text { (n) }\end{array}$ & $\begin{array}{c}\text { Egg-Based } \\
\text { IV } \\
\text { (n) }\end{array}$ & $\begin{array}{c}\text { Influenza } \\
\text { Related } \\
\text { Outcomes } \\
\text { (n) }\end{array}$ & $\begin{array}{l}\operatorname{arVE} \\
(\%)\end{array}$ & \multicolumn{2}{|c|}{$\operatorname{arVE~} 95 \% \mathrm{CI}$} & Funding \\
\hline Boikos 2020a [35] & $\begin{array}{c}2017- \\
2018\end{array}$ & $\begin{array}{c}\text { United } \\
\text { States }\end{array}$ & $\begin{array}{c}\text { Cohort- } \\
\text { retrospective }\end{array}$ & $\geq 65$ & Outpatient consultation & Codes & Moderate & 29,618 & 521 & 164,151 & 4808 & -7.3 & -51.6 & 24 & Seqirus \\
\hline Boikos 2020b [35] & $\begin{array}{c}2017- \\
2018\end{array}$ & $\begin{array}{l}\text { United } \\
\text { States }\end{array}$ & $\begin{array}{c}\text { Cohort- } \\
\text { retrospective }\end{array}$ & $18-64$ & Outpatient consultation & Codes & Moderate & 55,104 & 1069 & 693,014 & 10,021 & 26.8 & 14.1 & 37.6 & Seqirus \\
\hline Bruxvoort 2019a [28] & $\begin{array}{c}2017- \\
2018\end{array}$ & $\begin{array}{l}\text { United } \\
\text { States }\end{array}$ & Test-negative & $\geq 65$ & $\begin{array}{l}\text { Admission with } \\
\text { influenza }\end{array}$ & Lab-confirmed & Low & 157 & 25 & 3498 & 612 & 6 & -46 & 39 & Seqirus \\
\hline Divino 2020 [29] & $\begin{array}{c}2017- \\
2018\end{array}$ & $\begin{array}{l}\text { United } \\
\text { States }\end{array}$ & $\begin{array}{c}\text { Cohort- } \\
\text { retrospective }\end{array}$ & $18-64$ & $\begin{array}{c}\text { Admission \& } \\
\text { emergency room visits }\end{array}$ & Codes & Moderate & 499,156 & 976 & $1,730,403$ & 4053 & 13.1 & 6.8 & 19 & Seqirus \\
\hline Eick-Cost 2018 [54] & $\begin{array}{c}2017- \\
2018 \\
\end{array}$ & $\begin{array}{c}\text { Not } \\
\text { reported }\end{array}$ & Test-negative & $18-64$ & $\begin{array}{c}\text { Medical encounter with } \\
\text { influenza }\end{array}$ & Lab-confirmed & Moderate & 2467 & 506 & 3239 & 757 & 5 & -10 & 17 & $\begin{array}{c}\text { Defence } \\
\text { Health Agency } \\
\end{array}$ \\
\hline Izurieta 2019a [32] & $\begin{array}{c}2017- \\
2018\end{array}$ & $\begin{array}{l}\text { United } \\
\text { States }\end{array}$ & $\begin{array}{c}\text { Cohort- } \\
\text { retrospective }\end{array}$ & $\geq 65$ & Outpatient consultation & Codes & Low & 659,249 & 3299 & $1,863,654$ & 9607 & 5.7 & 1.9 & 9.4 & FDA \\
\hline Izurieta 2019b [32] & $\begin{array}{c}2017- \\
2018\end{array}$ & $\begin{array}{l}\text { United } \\
\text { States }\end{array}$ & $\begin{array}{c}\text { Cohort- } \\
\text { retrospective }\end{array}$ & $\geq 65$ & $\begin{array}{c}\text { Admission \& } \\
\text { emergency room visits }\end{array}$ & Codes & Low & 659,249 & 4370 & $1,863,654$ & 14,417 & 11 & 7.9 & 14 & FDA \\
\hline Klein 2020a [34] & $\begin{array}{c}2017- \\
2018 \\
\end{array}$ & $\begin{array}{l}\text { United } \\
\text { States }\end{array}$ & $\begin{array}{c}\text { Cohort- } \\
\text { retrospective }\end{array}$ & $18-64$ & $\begin{array}{c}\text { Medical encounter, } \\
\text { with influenza, A }\end{array}$ & Lab-confirmed & Low & 40,685 & . & 712,126 & . & -5.8 & -36.1 & 17.7 & DHHS \\
\hline Klein 2020b [34] & $\begin{array}{c}2017- \\
2018\end{array}$ & $\begin{array}{l}\text { United } \\
\text { States }\end{array}$ & $\begin{array}{c}\text { Cohort- } \\
\text { retrospective }\end{array}$ & $18-64$ & $\begin{array}{c}\text { Medical encounter, } \\
\text { with influenza, B }\end{array}$ & Lab-confirmed & Low & 40,685 & . & 712,126 & . & 21.4 & -7.3 & 42.4 & DHHS \\
\hline Martin 2020a [55] & $\begin{array}{c}2017- \\
2018\end{array}$ & $\begin{array}{c}\text { United } \\
\text { States }\end{array}$ & Test-negative & $\geq 18$ & $\begin{array}{l}\text { Admission with lab } \\
\text { confirmed A(H3N2) }\end{array}$ & Lab-confirmed & Low & 56 & 7 & 1459 & 248 & 24.9 & -78.8 & 68.5 & $\mathrm{CDC}, \mathrm{NIH}$ \\
\hline Martin 2020b [55] & $\begin{array}{c}2017- \\
2018 \\
\end{array}$ & $\begin{array}{l}\text { United } \\
\text { States }\end{array}$ & Test-negative & $\geq 18$ & $\begin{array}{c}\text { Admission with } \\
\text { B/Yamagata-lineage }\end{array}$ & Lab-confirmed & Low & 43 & 3 & 1135 & 83 & 1.8 & -254 & 72.8 & $\mathrm{CDC}, \mathrm{NIH}$ \\
\hline Martin 2020c [55] & $\begin{array}{c}2017- \\
2018\end{array}$ & $\begin{array}{l}\text { United } \\
\text { States }\end{array}$ & Test-negative & $\geq 18$ & $\begin{array}{l}\text { Admission with } \\
\text { influenza }\end{array}$ & Lab-confirmed & Low & 65 & 14 & 1676 & 399 & 8.5 & -75.9 & 52.3 & $\mathrm{CDC}, \mathrm{NIH}$ \\
\hline Boikos 2021c [57] & $\begin{array}{c}2018- \\
2019\end{array}$ & $\begin{array}{l}\text { United } \\
\text { States }\end{array}$ & $\begin{array}{c}\text { Cohort- } \\
\text { retrospective }\end{array}$ & $\geq 65$ & Medical encounter & Codes & Low & 517,639 & 6321 & 987,943 & 11,545 & -2.2 & -5.4 & 0.9 & Seqirus \\
\hline Boikos 2021d [57] & $\begin{array}{c}2018- \\
2019 \\
\end{array}$ & $\begin{array}{l}\text { United } \\
\text { States }\end{array}$ & $\begin{array}{c}\text { Cohort- } \\
\text { retrospective }\end{array}$ & $18-64$ & Medical encounter & Codes & Low & $1,529,189$ & 24,084 & $5,384,922$ & 87,113 & 6.5 & 5.2 & 7.9 & Seqirus \\
\hline Fu Tseng 2019a [52] & $\begin{array}{c}2018- \\
2019 \\
\end{array}$ & $\begin{array}{l}\text { United } \\
\text { States }\end{array}$ & Test-negative & $\geq 65$ & $\begin{array}{l}\text { Admission with } \\
\text { influenza A }\end{array}$ & Lab-confirmed & Low & 696 & 39 & 2773 & 146 & -6 & -54.3 & 28 & $\begin{array}{c}\text { Kaiser } \\
\text { Permanente }\end{array}$ \\
\hline
\end{tabular}




\begin{tabular}{|c|c|c|c|c|c|c|c|c|c|c|c|c|c|c|c|}
\hline Fu Tseng 2019b [52] & $\begin{array}{c}2018- \\
2019 \\
\end{array}$ & $\begin{array}{l}\text { United } \\
\text { States }\end{array}$ & Test-negative & $\geq 65$ & $\begin{array}{c}\text { Admission with } \\
\text { influenza }\end{array}$ & Lab-confirmed & Low & 696 & 39 & 2773 & 143 & -4 & -50.3 & 26 & $\begin{array}{c}\text { Kaiser } \\
\text { Permanente } \\
\end{array}$ \\
\hline Fu Tseng 2019c [52] & $\begin{array}{c}2018- \\
2019 \\
\end{array}$ & $\begin{array}{l}\text { United } \\
\text { States } \\
\end{array}$ & Test-negative & $\geq 65$ & $\begin{array}{c}\text { Admission with } \\
\text { influenza } \mathrm{A}(\mathrm{H} 1 \mathrm{~N} 1)\end{array}$ & Lab-confirmed & Low & 696 & 22 & 2773 & 65 & -32 & -117 & 20 & $\begin{array}{c}\text { Kaiser } \\
\text { Permanente } \\
\end{array}$ \\
\hline Fu Tseng 2019d [52] & $\begin{array}{c}2018- \\
2019 \\
\end{array}$ & $\begin{array}{l}\text { United } \\
\text { States }\end{array}$ & Test-negative & $\geq 65$ & $\begin{array}{c}\text { Admission with } \\
\text { influenza } \mathrm{A}(\mathrm{H} 3 \mathrm{~N} 2)\end{array}$ & Lab-confirmed & Low & 696 & 13 & 2773 & 52 & 6 & -75 & 49 & $\begin{array}{c}\text { Kaiser } \\
\text { Permanente }\end{array}$ \\
\hline Fu Tseng 2019e [52] & $\begin{array}{c}2018- \\
2019 \\
\end{array}$ & $\begin{array}{c}\text { United } \\
\text { States } \\
\end{array}$ & Test-negative & $\geq 65$ & $\begin{array}{c}\text { Admission with } \\
\text { influenza A untyped }\end{array}$ & Lab-confirmed & Low & 696 & 4 & 2773 & 26 & 36 & -86 & 78 & $\begin{array}{c}\text { Kaiser } \\
\text { Permanente }\end{array}$ \\
\hline Izurieta 2020a [33] & $\begin{array}{c}2018- \\
2019 \\
\end{array}$ & $\begin{array}{c}\text { United } \\
\text { States }\end{array}$ & $\begin{array}{c}\text { Cohort- } \\
\text { retrospective }\end{array}$ & $\geq 65$ & $\begin{array}{c}\text { Admission \& } \\
\text { emergency room visits }\end{array}$ & Codes & Low & 761,037 & 2330 & $1,454,340$ & 4582 & 2.5 & -2.4 & 7.3 & FDA \\
\hline Izurieta 2020b [33] & $\begin{array}{c}2018- \\
2019 \\
\end{array}$ & $\begin{array}{l}\text { United } \\
\text { States }\end{array}$ & $\begin{array}{c}\text { Cohort- } \\
\text { retrospective }\end{array}$ & $\geq 65$ & Admission & Codes & Low & 761,037 & 1426 & $1,454,340$ & 2790 & 4.4 & -1.9 & 10.3 & FDA \\
\hline $\begin{array}{c}\text { Krishnarajah } 2021 \\
{[53]} \\
\end{array}$ & $\begin{array}{c}2018- \\
2019 \\
\end{array}$ & $\begin{array}{c}\text { United } \\
\text { States } \\
\end{array}$ & $\begin{array}{c}\text { Cohort- } \\
\text { retrospective }\end{array}$ & 18-64 & $\begin{array}{c}\text { Admission \& } \\
\text { emergency room visits }\end{array}$ & Codes & Low & 590,705 & 768 & $2,223,435$ & 3113 & 4.9 & -2.8 & 12.1 & Seqirus \\
\hline Izurieta 2020c [56] & $\begin{array}{c}2019- \\
2020 \\
\end{array}$ & $\begin{array}{c}\text { United } \\
\text { States } \\
\end{array}$ & $\begin{array}{c}\text { Cohort- } \\
\text { retrospective }\end{array}$ & $\geq 65$ & $\begin{array}{c}\text { Admission \& } \\
\text { emergency room visits }\end{array}$ & Codes & Low & 824,264 & 2092 & $1,584,451$ & 3956 & 2.5 & -2.8 & 7.6 & FDA \\
\hline Izurieta 2020d [56] & $\begin{array}{c}2019- \\
2020 \\
\end{array}$ & $\begin{array}{l}\text { United } \\
\text { States } \\
\end{array}$ & $\begin{array}{c}\text { Cohort- } \\
\text { retrospective }\end{array}$ & $\geq 65$ & Admission & Codes & Low & 824,264 & 1255 & $1,584,451$ & 2309 & 1.3 & -5.7 & 7.9 & FDA \\
\hline
\end{tabular}

${ }^{*}$ All outcomes are either influenza related or with laboratory confirmed influenza, see next column, outcome definition method. ${ }^{+}$Codes: International Classification of Diseases, Tenth Revision, Clinical Modification codes: J09 Influenza due to certain identified influenza virus. J10 Influenza due to other identified influenza virus. J10.0 Influenza with pneumonia, other influenza virus identified. J10.1 Influenza with other respiratory manifestations, other influenza virus identified. J10.8 Influenza with other manifestations, other influenza virus identified. J11 Influenza, virus not identified. J11.0 Influenza with pneumonia, virus not identified. J11.1 Influenza with other respiratory manifestations, virus not identified. J11.8 Influenza with other manifestations, virus not identified. On the three studies by Izurieta et al. the code J12.9 Viral pneumonia, unspecified was added to define the outcomes. Laboratory confirmed outcomes: all Real time polymerase chain reactions. IV, influenza vaccine. arVE, adjusted relative vaccine effectiveness. CI confidence interval.

Table 2. Number of publications, results, mean number of subjects vaccinated, IRO cases, adjusted relative risk estimates, heterogeneity and test group differences after excluding mutually-dependent results*, by category of confounders or effect modifiers.

\begin{tabular}{|c|c|c|c|c|c|c|c|c|c|c|c|c|c|c|}
\hline \multirow[t]{2}{*}{ effect modifiers } & \multicolumn{2}{|c|}{ included } & \multicolumn{2}{|c|}{ Reported results } & \multirow{2}{*}{$\begin{array}{l}\text { Cell-cultured } \\
\qquad \text { IV } \\
\text { mean }\end{array}$} & IRO & \multirow{2}{*}{$\begin{array}{c}\text { Egg-based } \\
\text { IV } \\
\text { mean }\end{array}$} & \multirow{2}{*}{$\begin{array}{l}\text { IRO } \\
\text { mean } \\
\end{array}$} & \multirow[t]{2}{*}{ aRRף } & & & Heterogeneity & \multicolumn{2}{|c|}{$\begin{array}{r}\text { Test of group } \\
\text { differences }\end{array}$} \\
\hline & $n=12^{+}$ & $\%$ & $n=26$ & $\%$ & & mean & & & & & & $12(\%) \S$ & $\mathrm{Q}^{* *}$ & $\mathrm{p}$-value \\
\hline Season & & & & & & & & & & & & & 16.86 & $<0.001$ \\
\hline 2017-2018 & 7 & 58.3 & 14 & 53.8 & 188,995 & 1,111 & 686,936 & 4,483 & 0.89 & 0.86 & 0.91 & 0.01 & & \\
\hline 2018-2019 & 4 & 33.3 & 10 & 38.5 & 416,309 & 3,505 & $1,151,885$ & 10,958 & 0.97 & 0.93 & 1.02 & 79.15 & & \\
\hline 2019-2010 & 1 & 8.3 & 2 & 7.7 & 824,264 & 1,674 & $1,584,451$ & 3,133 & 0.98 & 0.92 & 1.02 & 0.00 & & \\
\hline
\end{tabular}




\begin{tabular}{|c|c|c|c|c|c|c|c|c|c|c|c|c|c|c|}
\hline Age group & & & & & & & & & & & & & 2.16 & 0.340 \\
\hline$>=18$ & 1 & 11.5 & 3 & 10.7 & 55 & 8 & 1,423 & 243 & 0.92 & 0.48 & 1.76 & 0.00 & & \\
\hline $18-64$ & 6 & 26.9 & 7 & 25.0 & 393,999 & 5,481 & $1,637,038$ & 21,011 & 0.91 & 0.85 & 0.97 & 72.11 & & \\
\hline$>=65$ & 7 & 61.5 & 16 & 57.1 & 356,212 & 1,519 & 802,583 & 3,991 & 0.97 & 0.92 & 1.02 & 78.23 & & \\
\hline $\begin{array}{l}\text { Study design } \\
\text { Cohort- }\end{array}$ & & & & & & & & & & & & & 0.11 & 0.740 \\
\hline Retrospective & 8 & 66.7 & 15 & 57.7 & 563,409 & 3,926 & $1,618,444$ & 12,821 & 0.94 & 0.89 & 0.98 & 89.21 & & \\
\hline Test-Negative & 4 & 33.3 & 11 & 42.3 & 584 & 63 & 2,563 & 270 & 0.96 & 0.85 & 1.08 & 0.00 & & \\
\hline Outcome setting & & & & & & & & & & & & & 1.78 & 0.410 \\
\hline Primary Care & 2 & 16.7 & 3 & 11.5 & 247,990 & 1,630 & 906,940 & 8,145 & 0.88 & 0.72 & 1.08 & 80.10 & & \\
\hline Hospital & 8 & 66.7 & 18 & 69.2 & 310,162 & 885 & 765,760 & 2,544 & 0.93 & 0.89 & 0.98 & 61.20 & & \\
\hline Both & 3 & 25.0 & 5 & 19.2 & 426,133 & 10,304 & $1,560,071$ & 33,138 & 0.98 & 0.92 & 1.04 & 85.13 & & \\
\hline Outcome & & & & & & & & & & & & & & \\
\hline determination & & & & & & & & & & & & & ככ. & 0.460 \\
\hline Laboratory & & & & & & & & & & & & & & \\
\hline confirmed & 5 & 41.7 & 13 & 50.0 & 6,753 & 63 & $111, / 27$ & 270 & 0.98 & 0.87 & 1.09 & 0.00 & & \\
\hline Clinical Codes & 7 & 58.3 & 13 & 50.0 & 643,828 & 3,926 & $1,757,878$ & 12,821 & 0.93 & 0.89 & 0.98 & 90.60 & & \\
\hline Funding & & & & & & & & & & & & & 0.36 & 0.550 \\
\hline Other & 7 & 58.3 & 18 & 69.2 & 290,879 & 1,122 & 728,565 & 2,996 & 0.95 & 0.90 & 1.00 & 57.05 & & \\
\hline Pharma & 5 & 41.7 & 8 & 30.8 & 402,715 & 4,223 & $1,398,836$ & 15,213 & 0.92 & 0.85 & 1.00 & 92.69 & & \\
\hline Risk of bias & & & & & & & & & & & & & 2.05 & 0.150 \\
\hline Low & 9 & 75.0 & 22 & 84.6 & 357,782 & 2,433 & 987,002 & 7,500 & 0.96 & 0.92 & 1.00 & 78.95 & & \\
\hline Moderate & 3 & 25 & 4 & 15.4 & 146,586 & 768 & 647,702 & 4,910 & 0.87 & 0.77 & 0.99 & 65.09 & & \\
\hline
\end{tabular}

IRO, influenza related outcome. IV, Influeza vaccine. aRR, adjusted relative risk of IRO comparig cell-cultured with egg-based vaccine: $<1$ favors cell culture vaccine. Cl, confidence interval.

* Excluded results: Bruxvoort 2019b; Izurieta 2019a; Izurieta 2019c; Klein 2020b; Martin 2020a; Martin 2020b; Fu Tseng 2019a; Fu Tseng 2019c; Fu Tseng 2019d; Fu Tseng 2019e, Izurieta 2020b, and Izurieta 2020d.

+ When totals are higher that 12 is because one study reports more that one result in the same age category.

I Adjusted relaive vaccine effectiveness estimated as $(1-\mathrm{aRR}) * 100$ 
§ Statistic for assessing heterogeneity. It estimates the proportion of variation between the effect sizes due to heterogeneity relative to the pure sampling variation. $12>50$ indicates substantial heterogeneity.

\section{** The $Q$ homogeneity test evaluates whether the effect sizes are the same across the results. We use the significance level $\geq 0.1$. The test does not estimate the magnitude of the heterogeneity.}


Two test-negative publications reported arVE results in preventing admissions with laboratory-confirmed A(H3N2) in the 2017-2018 season [28,55]. The point estimates of the two publications arVE results were non-significant, discordant, $-4 \%$ and to $24.9 \%$, and with overlapping intervals.

The previous two publications and two additional ones [28,34,54,55] also reported arVE for the 2017-2018 influenza season in preventing laboratory-confirmed outcomes with influenza overall, influenza A or B, the IROs were either admissions or medical encounters, one was in subjects aged 18 and over [55], two in subjects aged 18 to 64 [34,54] and one in subjects 65 and over [28]. Only one publication reported laboratoryconfirmed results on arVE in preventing laboratory-confirmed outcomes in the 2018-2019 influenza season [52].

After accounting for multiple-dependent results, we report the pooled results estimates by IRO determination method and other potential confounders or effect modifiers in Table 2.

Overall the pooled aRRs ranged from 0.87 to 0.98 fovoring the cell-cultured vaccine (pooled arVE range of $2 \%$ to $13 \%$ ). We observed substantial heterogeneity by season ( $p=$ 0.001 ). We could not reject the hypothesis of homogeneity in results by age-group ( $p=$ $0.340)$, study design $(p=0.740)$, outcome setting $(p=0.410)$, or funding $(p=0.550)$, and did not find evidence of differences between the pooled arVE results of laboratory-confirmed IRO compared to the pooled results obtained from code-ascertained IROs $(p=0.460)$ or risk of bias $(p=0.150)$.

\subsection{Heterogeneity}

We assessed heterogeneity by plotting the 26 retrieved results in a Galbraight plot (Figure 3). All results, except one (Boikos 2020b [35]), were inside the 95\% confidence region.

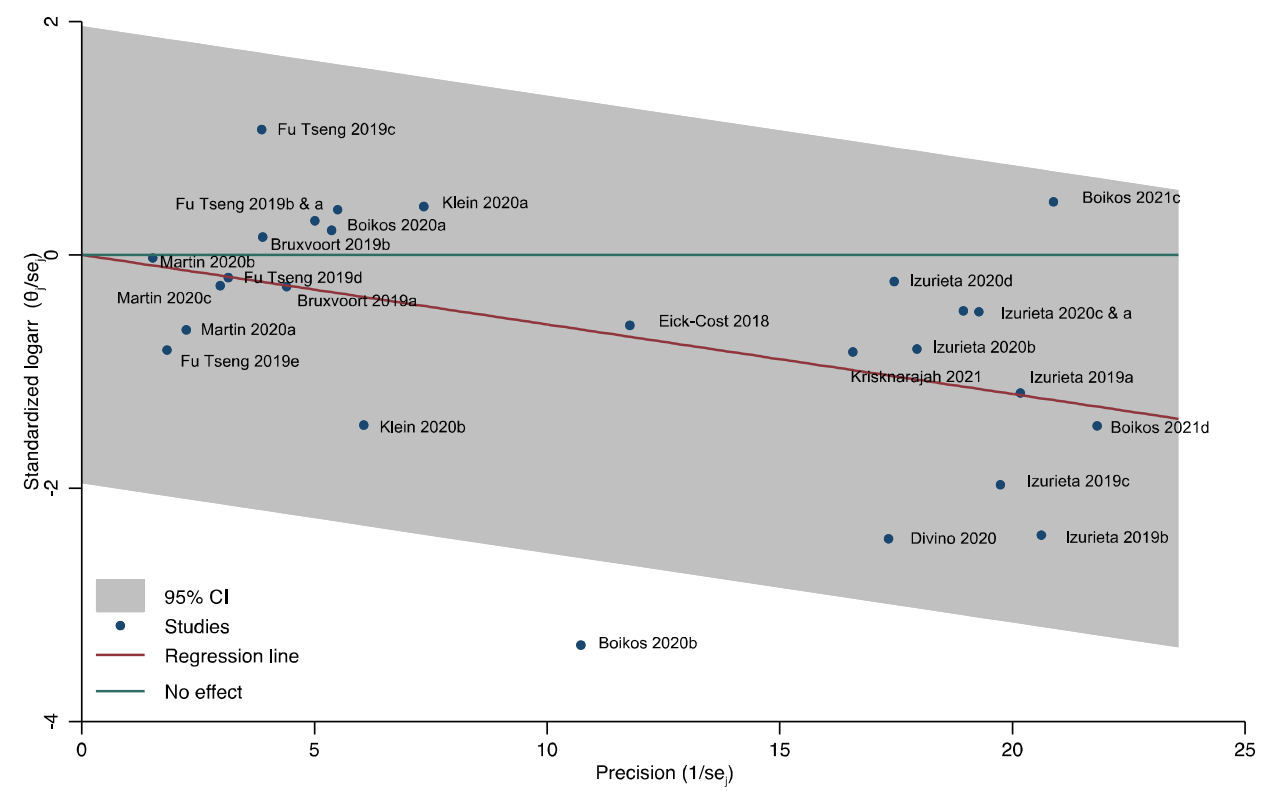

Figure 3. Heterogeneity and outliers among all included results. Relative vaccine effectiveness of cell - culture vs. eggbased vaccine in subjects $\geq 18$. 
We further assessed the heterogeneity with a forest-plot of the pooled results by season, age-group, outcome, study design, outcome determination method, funding and risk of bias (Figure 4). We restricted our analysis to not mutually-dependent results to avoid multiple contribution bias. We excluded results reported in only one study, such as those in subjects 18 and over [55] or the results for the 2019-2020 season [56]; finally, we also excluded the outlier result (Boikos 2020b [35]) identified in the Galbraith plot.

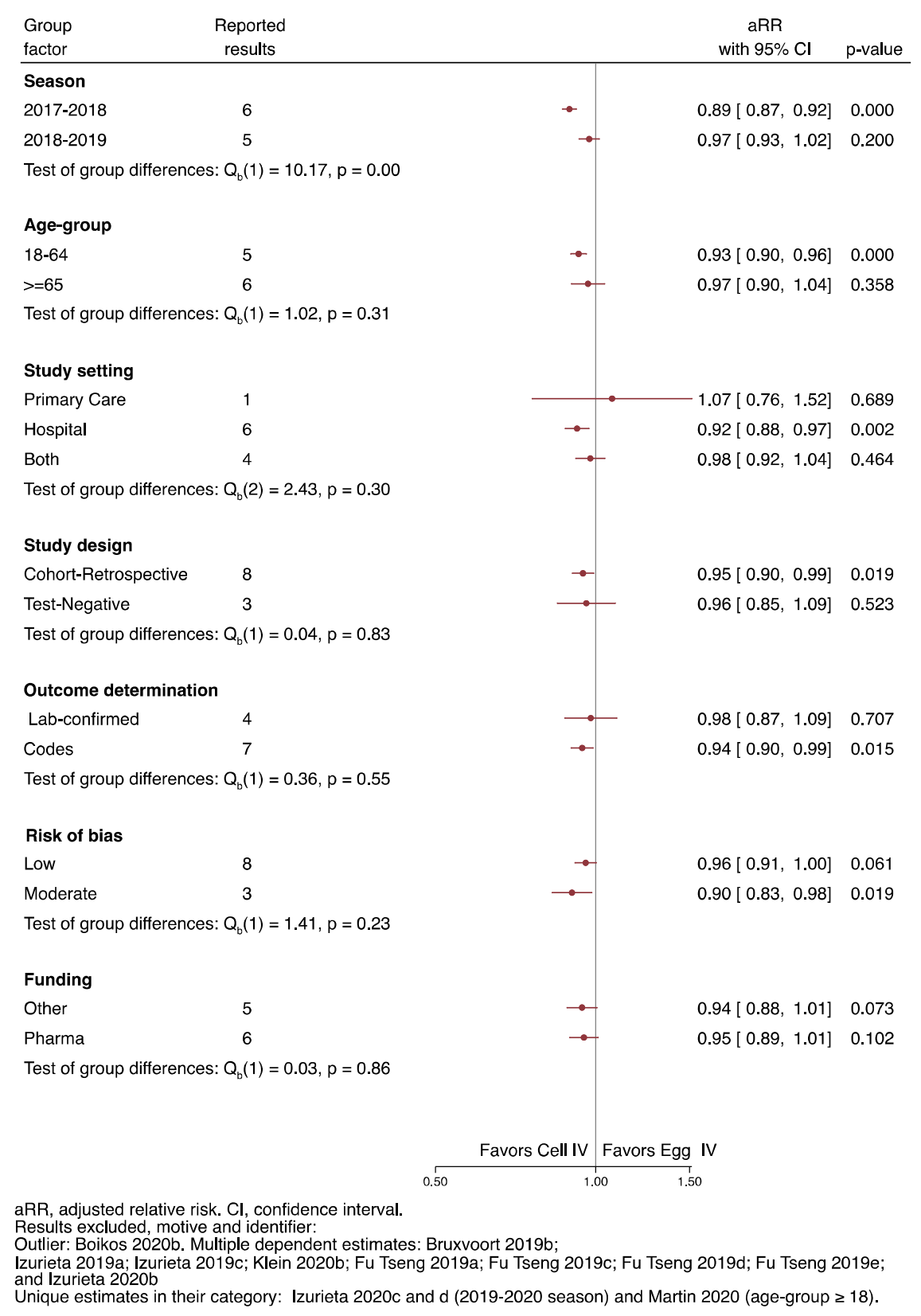

Figure 4. Heterogeneity of the results by season, age-group, study setting, study design, outcome determination method, risk of bias and funding.

After applying the above restrictions, we could reject homogeneity by season, with a pooled aRR for the season 2017-2018 of 0.89 (95\%CI 0.87 to 0.92 ), compared to 0.97 (0.931.02) for the 2018-2019 influenza season $(Q, p=0.00)$. 
We observed significant results favoring the cell-cultured vaccine in the 2017-2018 season, in the 18 to 64 age group $(0.93 ; 0.90$ to 0.96$)$, in preventing hospital-related IROs $(0.92 ; 0.88-0.97)$, in the cohort-retrospective studies $(0.95 ; 0.90-0.99)$, outcome derermination by code $(0.94 ; 0.90-0.99)$, and studies at moderate risk of bias $(0.90 ; 0.83-$ 0.98).

With the same restrictions, we did not observe that other clinical or methodological factors such as adjustment for previous health care contacts, underlying conditions, frailty, previous vaccination, calendar time, and vaccination date or statistical method of data analysis had an impact on the homogeneity of the results (Figure S1).

\subsection{Publication Bias}

We did not detect evidence of publication bias or reporting asymmetry when considering the 26 included results (Egger test $p$-value of 0.9926; Figure S2). We performed the same statistical and graphical analysis by season, age group, study design, reported outcome, outcome determination method, funding and risk of bias and did not obtain evidence of plot asymmetry by the Egger test for any of the above factors, but some patterns emerged in the by factor funnel plots (Figure S3). We observed a homogenous distribution of retrieved results in the 2017-2018 and 2018-2019 seasons, but only two results in the 2019-2020 season [56]. We did not observe asymmetric distribution for the results in subjects $\geq 65$, test-negative studies, influenza-related hospital outcomes, laboratory-confirmed results, non-pharma funded studies and results obtained in studies at low RoB. There was a lack of small studies results for the 18-64 age group; primary care and medical encounters (defined as contacts with either primary or hospital care) IRO; in the pharma funded studies, and the cohort-retrospective studies.

\subsection{Meta-Analysis of the Published Relative Vaccine Effectiveness Results}

After considering our predefined criteria for meta-analysis, we estimated the pooled arVE results for laboratory-confirmed IROs with influenza [28,34,54,55], (Figure 5a) and the pooled arVE regardless of the IRO determination method [28,29,32,34,35,54], in this instance, stratified by season and age group (Figure $5 b, c$ ).

\subsubsection{Adjusted Relative Vaccine Effectiveness in Preventing Laboratory-Confirmed IRO}

When we pooled the results of the influenza laboratory-confirmed results obtained in the 2017-2018 season [28,34,54,55], we estimated a pooled aRR of 0.97 (IC95\% of 0.86 to 1.09), with an overall heterogeneity $\left(\mathrm{I}^{2}\right)$ of $0.0 \%$, and homogenous results among publications (Cochran's Q, $p=0.90$ ); with a non-significant adjusted arVE of 3\% ( $-9 \%$ to $14 \%$ ) favoring the cell-cultured influenza vaccine compared to the egg-based influenza vaccine in preventing laboratory-confirmed IRO.

\subsubsection{Adjusted Relative Vaccine Effectiveness in Preventing any IRO}

The overall aRR in preventing any IRO in the 2017-2018 influenza season was 0.89 $(0.87 ; 0.92)$ (Figure $5 b)$, with an overall heterogeneity of $0.0 \%$, with homogenous results across age groups (Cochran's $Q, p=0.64)$. By age, the aRR was $0.91(0.83-1.00)$ in the 1864 age group $\left(\mathrm{I}^{2}, 35.85 \%\right)$, with no differences among results of the included publications, $\mathrm{Q}, p=0.22$, and $0.89(0.86-0.92)$ in the $\geq 65$ age group $\left(\mathrm{I}^{2}=0.0 \%\right)$, with no differences among results of the included publications, $\mathrm{Q}, p=0.58$.

Overall, it translated to a significant arVE of $11 \%(8 \%$ to $13 \%)$ in preventing IRO favoring the cell-cultured versus the egg-based vaccine in the 2017-2018 season. 


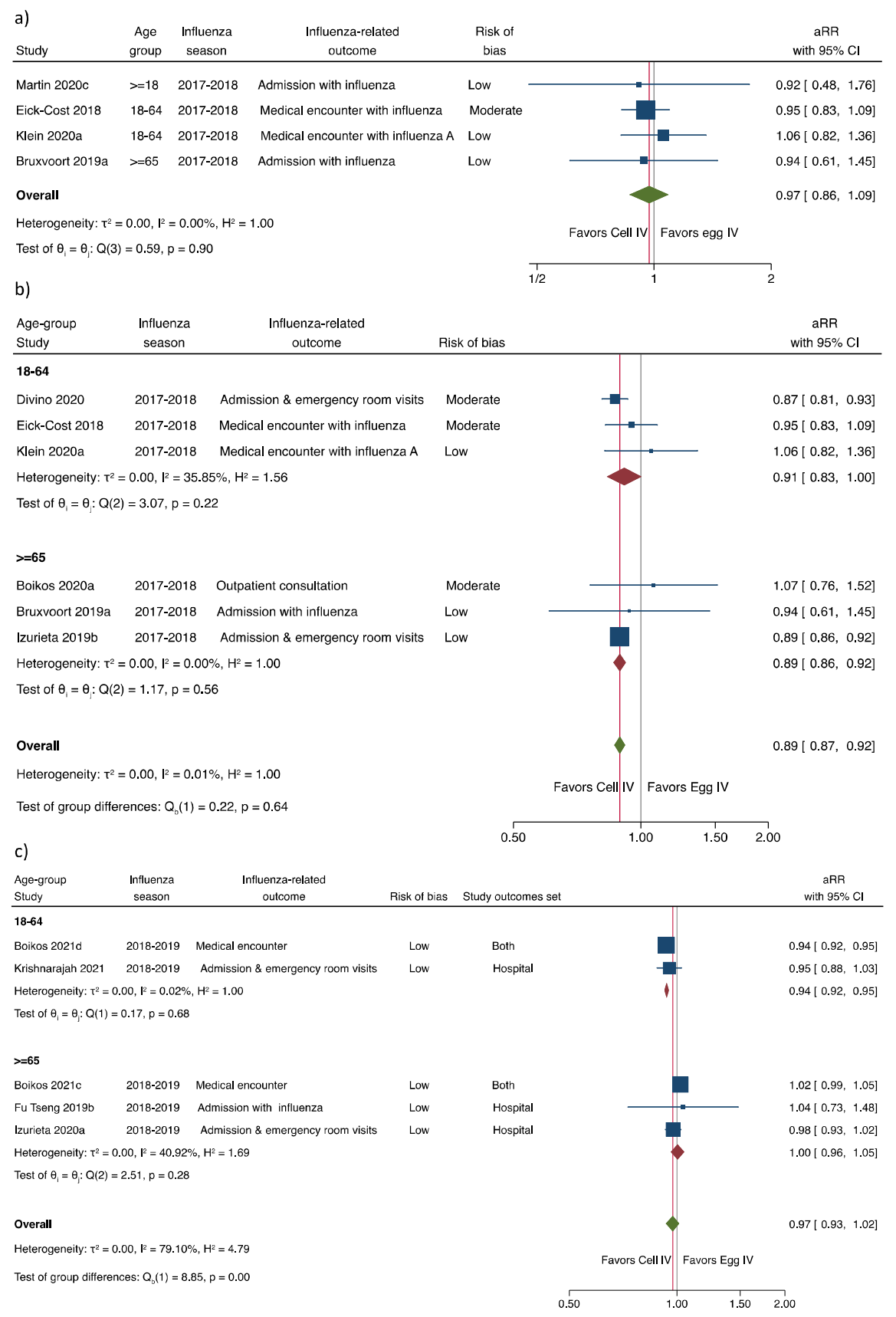

Random-effects REML mode

aRR, adjusted relative risk. $\mathrm{Cl}$, confidence interval

Cell IV, seed cell-cultured influenza vaccine. Egg IV, egg-based influenza vaccine

adjusted relative vaccine effectiveness $=(1-\mathrm{aRR}) * 100 ")$

Figure 5. Adjusted relative risk of laboratory-confirmed influenza related outcomes comparing cell-cultured versus egg based influenza vaccines (a) Laboratory confirmed outcomes, 2017 - 2018 influenza season: (b) Code confirmed and laboratory confirmed outcomes, 2017 - 2018 influenza season; (c) Code confirmed and laboratory confirmed outcomes,2018 - 2019 influenza season.

There were five results for the 2018-2019 season [33,52,53,57] with significant heterogeneity $\mathrm{I}^{2}=79.10 \%$ and a Cochran's $\mathrm{Q} p=0.00$ (Figure $5 \mathrm{c}$ ). The overall result was an 
aRR of 0.97 (0.93-1.02). By age group, the aRR was $0.94(0.92-0.95), \mathrm{I}^{2} 0.02 \%$, and Cochran's $\mathrm{Q}, p=0.68$ in those aged 18-64, and $1.00(0.96-1.05), \mathrm{I}^{2} 40.92 \%$, and Cochran's $\mathrm{Q}, p=0.28$ in those aged 65 and over. Overall, the arVE of 3\% (-2\%, 7\%), favoring the cell-culture vaccine with a significant result (6\%; IC95\% 5\% to 8\%) for the 18-64 age-group.

\subsection{Sensitivity Analysis}

In the non-parametric trim and fill analysis restricted to the 2017-2018 season, the predicted aRR interval with the observed and imputed studies was $0.89(0.86-0.91)$, with three new imputed studies all favoring the cell-culture vaccine (Figure S4). When repeating the same analysis, including the excluded multiple dependent results, we obtained a similar effect with an estimated aRR of 0.90 (0.87-0.93) and two new imputed results (Figure S5). The trim and fill analysis for the 2018-2019 season resulted in one imputed non-retrieved small-size study favoring the cell-cultured vaccine with no differences in the reported and the imputed results (data not shown).

We estimated the impact of excluding result by result in the influenza seasons with enough results (2017-2018 and 2018-2019) and did not observe significant differences with the results contained in the estimated overall confidence interval for each season (Table S2).

Finally, we studied the impact of the outlier result (Boikos 2020b [35]) in the estimates and heterogeneity of the 18-64 age group and overall results in the 2017-2018 influenza season. The aRR estimate was now of $0.88(0.77,1.00)$ for the $18-64$ age group and 0.89 $(0.86,0.91)$ for the overall estimate, with an $\mathrm{I}^{2}$ of $70.46 \%$ and $0 \%$, compared with the aRR of $0.91(0.83-1.00)$ and $0.89(0.87-0.92)$ and the heterogeneity $\left(\mathrm{I}^{2}\right)$ of $35.85 \%$ and $0 \%$, when we excluded this result as we show in the Figure $5 b$, resulting in a more precise estimate with lower heterogeneity for the 18-64 age-group, but with minor impact on the overall estimate.

\section{Discussion}

We identified 12 publications of non-randomised intervention studies that reported rVE of seed-cell ccIV compared to eIV in preventing IROs in the 2017-2018, 2018-2019 and 2019-2020 influenza seasons. The main source of heterogeneity on arVE estimates was the influenza season. We identified only three non-mutually dependent results on the arVE of ccIV compared to eIV in preventing A(H3N2) IRO, two in the 2017-2018 influenza season and one in the 2018-2019 influenza season. Following our secondary goal, we identified four publications that reported four homogenous laboratory-confirmed results obtained in different age groups in the 2017-2018 influenza season. The pooled arVE of these four results favored ccIV but was nonsignificant and with a broad confidence interval.

Finally, we identified six publications with six non mutually dependent, homogenous, with low heterogeneity, arVE results, three were real-world evidence studies, and three were laboratory-confirmed outcome studies. The pooled evidence was of the significant advantage of ccIV compared to eIV in preventing IRO in the 2017-2018 influenza season, regardless of IRO, outcome determination, setting or age group.

The results were null arVE for the 2018-2019 season, with substantial heterogeneity and strong evidence supporting age as a significant heterogeneity driver.

\subsection{Interpretation and Validity}

Overall our findings on homogeneity and heterogeneity by study characteristics are consistent with the existing evidence [58]. Others have explored and concluded the similarity of influenza vaccine effectiveness estimates obtained in primary care and inpatient settings, concluding that "no differences in VE estimates between inpatient and outpatient settings by studies using the test-negative design". Regarding the age effect, a previous systematic review reported similar estimates by age group by type or subtype of influenza virus, with the variability by age in vaccine effectiveness estimates explained 
mainly by the different magnitude of influenza vaccine effectiveness by influenza virus type or subtype [11,12]. Finally, other authors have described the modifying effect of previous exposure by the birth cohort that may result in a negative interaction between vaccination with an unmatched strain and imprinted immunity [59]. This situation has been proposed to explain the age variability of estimated influenza vaccine effectiveness during the mixed 2018-2019 season [60].

While all the described evidence on homogeneity or heterogeneity has been obtained from tests-negative studies, we have additionally observed overall homogeneity in the arVE estimates obtained from low to moderate RoB tests-negative and retrospectivecohort RWE studies, an observation that has to be validated or rejected as more evidence accumulates from real-world data retrospective-cohorts studies in future influenza seasons.

We would expect the benefits of ccIV compared to eIV in seasons with limited antigenic drift and egg adaptation in the egg-derived vaccine strains [14,61]. This was the situation in the 2017-2018 season, A(H3N2) was the only cell-based strain in the ccIV vaccine, $\mathrm{A}(\mathrm{H} 3 \mathrm{~N} 2)$ was predominant, the vaccine strain was well matched with the circulating A(H3N2) strain, and egg adaptation occurred [62]. By contrast, the 2018-2019 influenza season was mixed, with A(H1N1)pdm09 accounting for $48 \%$ of subtyped strains and $\mathrm{A}(\mathrm{H} 3 \mathrm{~N} 2)$ for $49 \%[63]$. Neither egg adaptation nor drift was observed for the $\mathrm{A}(\mathrm{H} 1 \mathrm{~N} 1) \mathrm{pdm} 09$, but drift was observed in the $\mathrm{A}(\mathrm{H} 3 \mathrm{~N} 2)$, and similarity between circulating and vaccine strains was notably poorer for both the egg- and cell-based vaccine $\mathrm{A}(\mathrm{H} 3 \mathrm{~N} 2)$ viruses [64].

All in all, the mentioned influenza seasons characteristics are consistent with our meta-analysis results of a significant arVE favoring the ccIV in the 2017-2018 season and the no effect in the 2018-2019 season. Regarding the 2018-2019 season results, we observed overall significant heterogeneity and a non-homogenous effect by age. We must stress, nevertheless, that our overall pooled result was of no arVE in preventing IRO, in line with the fact that in the U.S. 2018-2019 influenza season, no advantage over the ccIV over the eIV was likely, as the A(H1N1)pdm09 strain in the 2018-2019 ccIV vaccine was egg-derived, and that the age-specific low estimates were reported in the U.S. for the 20182019 season in preventing $\mathrm{A}(\mathrm{H} 3 \mathrm{~N} 2)$, but not in preventing $\mathrm{A}(\mathrm{H} 1 \mathrm{~N} 1)$ pdm09 IROs [63], a fact that could explain the observed age non-homogenous results. The results in the 20182019 (and the only study in the 2019-2020) do reinforce the observation in the 2017-2018 season of a favourable impact, compared to the egg-based vaccine, of the seed-cell-based vaccine. When there is no mismatch between the vaccine and the circulating strains, egg adaptation has a negative impact. In the presence of a mismatch, it is the mismatch the main driver to low vaccine effectiveness.

\subsection{Limitations}

Caution is advisable in interpreting the meta-analysis of evidence from observational studies, given the risk of robust and precise but biased estimates [65]. We collected results for only three seasons, and in the 2019-2020 season, we identified only one study. This evidence availability will not change soon, as 2020, 2020-2021, 2021, South or North hemisphere influenza seasons have been absent, and the 2021-2022 influenza season is at the time of writing a question mark. In addition to the limited number of seasons, we must add the limitations of the methods in the included studies and our approach and restrictions to analyse the available evidence.

The laboratory-confirmed test-negative studies' sources of bias reside in their small sample sizes and the limited virus subtyping. In addition to lack of precision and typing specificity, the poor information on how the subjects were enrolled is a critical point to judge the quality of test-negative studies, even when comparing vaccinated with vaccinated. In the absence of incidence density sampling or a well-run systematic recruitment process, critical selection bias cannot be discarded, jeopardizing the reliability 
of the retrieved test-negative studies, in which that information was usually poorly reported.

The main potential sources of bias in real-world evidence (RWE) studies are exposure determination, lack of information on confounders, outcome determination, proper adjustment and analysis. For the retrieved and recent RWE studies, exposure determination and information on confounders was adequate. The modelling and adjustment of real-world evidence studies have evolved significantly year by year. The use of propensity scores, inverse probability of treatment weighting, adjustment by calendar time and geographic region, and the use of Poisson regression provides a sound analytical framework. Nevertheless, we found a gap in reporting the number of subjects by group and the number of missing subjects by each of the analysed groups. In some of the studies, we missed calendar time adjustment and proper time-person analysis approaches. An additional weakness of RWE thus far is the specificity in the determination of IRO by clinical codes. Although, this should result in a non-differential classification bias and thus could only have an impact of a regression to the null on the arVE estimates. Supporting our argumentation and the overall results is, first, that it has been argued that when comparing observational study designs, imperfect specificity tends to under-estimate true vaccine effectiveness, but were similar across designs "except if fairly extreme inputs were used" [66], and, second, the good correlation between coding and actual influenza [67].

Regarding our approach, the judgments on RoB are, although well structured, qualitative and subject to researcher bias. In the homogeneity and heterogeneity analysis, our final attribution of the RoB category was not a significant effect modifier. We also decided not to include estimates in populations with high-risk conditions or by risk conditions. We argue that a focused, systematic review on special populations should be performed, and in our approach, the interest rested in the confounding and adjustment by underlying conditions. Moreover, analysis by high-risk conditions was reported only in few publications (Table S1). We also decided to exclude mutually dependent results to avoid the overweighting of the results of the same exposure-population experiences on the results. The sensitivity and specificity of the statistical $Q$ homogeneity test have been put into question, as in the presence of a small number of results, the $Q$ test lacks the power to identify non-homogeneity, as could be the case for $\operatorname{RoB}(p=0.15)$ due to the low number of results and studies classified of moderate RoB. In the presence of a large number of results, it offers false-positive results. And some authors counsel against it and prefer the $\mathrm{I}^{2}$ parameter [68]. We opted to report both as our database was in the middle of these two situations, but applying a conservative significance level alpha $\geq 0.1$ instead of the conventional alpha $\geq 0.05$ for the interpretation of the $Q$ test to accept homogeneity.

\subsection{Strengths}

We followed the PRISMA guidelines for the study design, retrieval, selection, RoB analysis, reporting and analysis, with a detailed description of the included publications, homogeneity, heterogeneity, publication bias and sensitivity analysis, followed by a conservative random approach analysis. We performed the quantitative analysis only when homogenous, with low heterogeneity, and sufficient results were available. Accordingly, we did not pool results in the presence of heterogeneity, such as pooling of matched and mismatched seasons, as, in the current situation, the season was a clear modifying factor and pooling inadequate. In this scenario, the most relevant information is provided in the stratified by season analysis. We restricted our systematic review to the whole cell-derived vaccines to exclude the question of strain egg-adaptation. Although we included the absolute numbers of subjects by category in all but one of the included publications, we used only adjusted results. Here we agree with other authors that argue on the usefulness of unadjusted results [69].

\section{Conclusions}


Our systematic review provides low to moderate evidence supporting the ccIV advantage in preventing $\mathrm{A}(\mathrm{H} 3 \mathrm{~N} 2)$ related IROs compared to eIV in well-matched $\mathrm{A}(\mathrm{H} 3 \mathrm{~N} 2)$ predominant influenza season. Supports the use of well-powered real-world evidence studies to provide timely real-world evidence on the comparative effectiveness of different influenza vaccines in preventing relevant IRO. Mainly, we have collected evidence that in the presence of low risk of bias, the results are homogenous across settings, outcome determination methods, and study design.

Supplementary Materials: The following are available online at www.mdpi.com/xxx/s1, Table S1. Studies reporting relative vaccine effectiveness of cell-derived influenza seasonal vaccines compared to egg-derived influenza seasonal vaccines, after the exclusion of duplicates. Table S2. Leave one out analysis, 2017-2018 and 2018-2019. Random-effects model Method: REML. Figure S1. Other confounders contribution to heterogeneity. Figure S2. Funnel plot of effect estimates of the relative vaccine effectiveness of cell-culture vs. egg-culture influenza vaccines in preventing influenza related outcomes in subjects $\geq 18$. Figure S3. Graphical and statistical bias analysis by season, age group, study design, reportedoutcome, outcome determination, funding and risk of bias. Figure S4. Non-parametric trim and fill analysis restricted to the 2017-2018 season Multipledependent and outlier results excluded. Figure S5. Non-parametric trim and fill analysis, 2017-2018 season, with multiple-dependent and outlier results. PRISMA checklist

Author Contributions: Conceptualization, J.P.-B.; methodology, J.P.-B., S.T.-G.; P.P.-R., J.M.E.-B.; formal analysis, J.P.-B.; investigation, J.P.-B., P.P.-R., S.T.-G., J.M.E.-B.; data curation, J.P.-B., P.P.-R..; writing-original draft preparation, J.P.-B.; writing-review and editing, J.P.-B., S.T.-G., P.P.-R., J.M.E.-B. All authors have read and agreed to the published version of the manuscript.

Funding: This work was supported by Seqirus Spain, S.L. The funder of the study had no role in the study design, data collection, data analysis, data interpretation, or writing of the report.

Institutional Review Board Statement: Not applicable.

Informed Consent Statement: Not applicable.

Data Availability Statement: The data presented in this study is available in the manuscript and Supplementary Materials.

Conflicts of Interest: JPB has received honoraria for academic activities from Sanofi Pasteur and Seqirus, Advisory board membership from Seqirus, research funding from Seqirus. Other not remunerated research activities with Sanofi Pasteur, Seqirus and BioNTech. ST has received honoraria for academic activities from GSK, Sanofi Pasteur, AstraZeneca and Seqirus. PPR has received honoraria for academic activities from Seqirus. JMEB has received honoraria for academic activities from GSK, Pfizer, Sanofi Pasteur and Seqirus. The funders had no role in the design of the study; in the collection, analyses, or interpretation of data; in the writing of the manuscript, or in the decision to publish the results. All the authors had access to the raw data and had final responsibility for the decision to submit for publication.

\section{References}

1. Troeger, C.E.; Blacker, B.F.; Khalil, I.A.; Zimsen, S.R.M.; Albertson, S.B.; Abate, D.; Abdela, J.; Adhikari, T.B.; Aghayan, S.; Agrawal, S.; et al. Mortality, morbidity, and hospitalisations due to influenza lower respiratory tract infections, 2017: an analysis for the Global Burden of Disease Study 2017. Lancet Respir. Med. 2019, 7, 69-89, https://doi.org/10.1016/s2213-2600(18)30496-x.

2. A Walker, T.; Waite, B.; Thompson, M.G.; McArthur, C.; Wong, C.; Baker, M.G.; Wood, T.; Haubrock, J.; Roberts, S.; Gross, D.K.; et al. Risk of Severe Influenza Among Adults With Chronic Medical Conditions. J. Infect. Dis. 2019, 221, 183-190, https://doi.org/10.1093/infdis/jiz570.

3. Arevalo, P.; McLean, H.Q.; A Belongia, E.; Cobey, S. Author response: Earliest infections predict the age distribution of seasonal influenza A cases. 2020, https://doi.org/10.7554/elife.50060.sa2.

4. Van Kerkhove, M.D.; Vandemaele, K.A.H.; Shinde, V.; Jaramillo-Gutierrez, G.; Koukounari, A.; Donnelly, C.A.; Carlino, L.O.; Owen, R.; Paterson, B.; Pelletier, L.; et al. Risk Factors for Severe Outcomes following 2009 Influenza A (H1N1) Infection: A Global Pooled Analysis. PLoS Med. 2011, 8, e1001053, https://doi.org/10.1371/journal.pmed.1001053.

5. Skowronski, D.M.; Chambers, C.; De Serres, G.; Sabaiduc, S.; Winter, A.-L.; Dickinson, J.; Gubbay, J.B.; Fonseca, K.; Drews, S.J.; Charest, H.; et al. Age-Related Differences in Influenza B Infection by Lineage in a Community-Based Sentinel System, 20102011 to 2015-2016, Canada. J. Infect. Dis. 2017, 216, 697-702, https://doi.org/10.1093/infdis/jix393. 
6. Song, J.Y.; Noh, J.Y.; Lee, J.S.; Wie, S.-H.; Kim, Y.K.; Lee, J.; Jeong, H.W.; Kim, S.W.; Lee, S.H.; Park, K.-H.; et al. Effectiveness of repeated influenza vaccination among the elderly population with high annual vaccine uptake rates during the three consecutive A/H3N2 epidemics. Vaccine 2019, 38, 318-322, https://doi.org/10.1016/j.vaccine.2019.10.012.

7. Lafond, K.E.; Porter, R.M.; Whaley, M.J.; Suizan, Z.; Ran, Z.; Aleem, M.A.; Thapa, B.; Sar, B.; Proschle, V.S.; Peng, Z.; et al. Global burden of influenza-associated lower respiratory tract infections and hospitalizations among adults: A systematic review and meta-analysis. PLoS Med. 2021, 18, e1003550, https://doi.org/10.1371/journal.pmed.1003550.

8. Paget, J.; Spreeuwenberg, P.; Charu, V.; Taylor, R.J.; Iuliano, A.D.; Bresee, J.; Simonsen, L.; Viboud, C.; Global Seasonal InfluenzaAssociated Mortality Collaborator Network and GLaMOR Collaborating Teams. Global mortality associated with seasonal influenza epidemics: New burden estimates and predictors from the GLaMOR Project. J. Glob. Health 2019, 9, 020421, doi:10.7189/jogh.09.020421.

9. Iuliano, A.D.; Roguski, K.M.; Chang, H.H.; Muscatello, D.J.; Palekar, R.; Tempia, S.; Cohen, C.; Gran, J.M.; Schanzer, D.; Cowling, B.J.; et al. Estimates of global seasonal influenza-associated respiratory mortality: a modelling study. Lancet 2018, 391, 1285-1300, doi:10.1016/s0140-6736(17)33293-2.

10. Hardelid, P.; Pebody, R.; Andrews, N. Mortality caused by influenza and respiratory syncytial virus by age group in England and Wales 1999-2010. Influ. Other Respir. Viruses 2012, 7, 35-45, https://doi.org/10.1111/j.1750-2659.2012.00345.x.

11. A Belongia, E.; Simpson, M.D.; King, J.; E Sundaram, M.; Kelley, N.S.; Osterholm, M.T.; McLean, H.Q. Variable influenza vaccine effectiveness by subtype: a systematic review and meta-analysis of test-negative design studies. Lancet Infect. Dis. 2016, 16, 942951, https://doi.org/10.1016/s1473-3099(16)00129-8.

12. Belongia, E.; McLean, H.Q. Influenza Vaccine Effectiveness: Defining the H3N2 Problem. Clin. Infect. Dis. 2019, 69, 1817-1823, https://doi.org/10.1093/cid/ciz411.

13. Skowronski, D.M.; Janjua, N.; De Serres, G.; Sabaiduc, S.; Eshaghi, A.; Dickinson, J.; Fonseca, K.; Winter, A.-L.; Gubbay, J.B.; Krajden, M.; et al. Low 2012-13 Influenza Vaccine Effectiveness Associated with Mutation in the Egg-Adapted H3N2 Vaccine Strain Not Antigenic Drift in Circulating Viruses. PLOS ONE 2014, 9, e92153, https://doi.org/10.1371/journal.pone.0092153.

14. Wu, N.C.; Zost, S.J.; Thompson, A.J.; Oyen, D.; Nycholat, C.M.; McBride, R.; Paulson, J.C.; Hensley, S.E.; Wilson, I.A. A structural explanation for the low effectiveness of the seasonal influenza H3N2 vaccine. PLOS Pathog. 2017, 13, e1006682, https://doi.org/10.1371/journal.ppat.1006682.

15. Gerdil, C. The annual production cycle for influenza vaccine. Vaccine 2003, 21, 1776-1779, https://doi.org/10.1016/s0264410x(03)00071-9.

16. Laurent, P.E.; Bonnet, S.; Alchas, P.; Regolini, P.; Mikszta, J.A.; Pettis, R.; Harvey, N.G. Evaluation of the clinical performance of a new intradermal vaccine administration technique and associated delivery system. Vaccine 2007, 25, 8833-8842, https://doi.org/10.1016/j.vaccine.2007.10.020.

17. Del Giudice, G.; Rappuoli, R.; Didierlaurent, A.M. Correlates of adjuvanticity: A review on adjuvants in licensed vaccines. Semin. Immunol. 2018, 39, 14-21, https://doi.org/10.1016/j.smim.2018.05.001.

18. Treanor, J.J. New approaches to influenza vaccine-High doses, neuraminidase vaccines, alternative substrates, and new adjuvants. Infect. Med. 1998, 15, 487-492.

19. DiazGranados, C.A.; Dunning, A.J.; Kimmel, M.; Kirby, D.; Treanor, J.; Collins, A.; Pollak, R.; Christoff, J.; Earl, J.; Landolfi, V.; et al. Efficacy of High-Dose versus Standard-Dose Influenza Vaccine in Older Adults. N. Engl. J. Med. 2014, 371, 635-645, doi:10.1056/nejmoa1315727.

20. Cox, M.M.; Hashimoto, Y. A fast track influenza virus vaccine produced in insect cells. J. Invertebr. Pathol. 2011, 107, S31-S41, https://doi.org/10.1016/j.jip.2011.05.003.

21. Milián, E.; Kamen, A.A. Current and Emerging Cell Culture Manufacturing Technologies for Influenza Vaccines. BioMed Res. Int. 2015, 2015, 1-11, https://doi.org/10.1155/2015/504831.

22. Galli, C.; Orsi, A.; Pariani, E.; Lai, P.L.; Guarona, G.; Pellegrinelli, L.; Ebranati, E.; Icardi, G.; Panatto, D. In-depth phylogenetic analysis of the hemagglutinin gene of influenza A(H3N2) viruses circulating during the 2016-2017 season revealed egg-adaptive mutations of vaccine strains. Expert Rev. Vaccines 2020, 19, 115-122, https://doi.org/10.1080/14760584.2020.1709827.

23. Centers for Disease Control and Prevention National Center for Immunization and Respiratory Diseases (NCIRD). How Influenza (Flu) Vaccines Are Made n.d. Available online: https://www.cdc.gov/flu/prevent/how-fluvaccine-made.htm (accessed on 13 March 2020).

24. Barr, I.G.; Donis, R.O.; Katz, J.M.; McCauley, J.W.; Odagiri, T.; Trusheim, H.; Tsai, T.F.; Wentworth, D.E. Cell culture-derived influenza vaccines in the severe 2017-2018 epidemic season: a step towards improved influenza vaccine effectiveness. $n p j$ Vaccines 2018, 3, 44, https://doi.org/10.1038/s41541-018-0079-z.

25. Grohskopf, L.A.; Sokolow, L.Z.; Broder, K.R.; Walter, E.B.; Fry, A.M.; Jernigan, D.B. Prevention and Control of Seasonal Influenza with Vaccines: Recommendations of the Advisory Committee on Immunization Practices-United States, 2018-19 Influenza Season. MMWR. Recomm. Rep. 2018, 67, 1-20, https://doi.org/10.15585/mmwr.rr6703a1.

26. European Medicines Agency. Flucelvax Tetra n.d. https:/www.ema.europa.eu/en/medicines/human/EPAR/flucelvax-tetra (accessed on 12 March 2021).

27. European Centre for Disease Prevention and Control. Systematic review of the efficacy, effectiveness and safety of newer and enhanced seasonal influenza vaccines for the prevention of laboratory-confirmed influenza in individuals aged 18 years and over. European Centre for Disease Prevention and Control: Stockholm, Sweden, 2020. https://doi.org/10.2900/751620. 
28. Bruxvoort, K.J.; Luo, Y.; Ackerson, B.; Tanenbaum, H.; Sy, L.S.; Gandhi, A.; Tseng, H.F. Comparison of vaccine effectiveness against influenza hospitalization of cell-based and egg-based influenza vaccines, 2017-2018. Vaccine 2019, 37, 5807-5811, https://doi.org/10.1016/j.vaccine.2019.08.024.

29. Divino, V.; Krishnarajah, G.; Pelton, S.I.; Mould-Quevedo, J.; Anupindi, V.R.; DeKoven, M.; Postma, M.J. A real-world study evaluating the relative vaccine effectiveness of a cell-based quadrivalent influenza vaccine compared to egg-based quadrivalent influenza vaccine in the US during the 2017-18 influenza season. Vaccine 2020, 38, 6334-6343, https://doi.org/10.1016/j.vaccine.2020.07.023.

30. Pelton, S.; Postma, M.; Divino, V.; Mould-Quevedo, J.; DeKoven, M.; Krishnarajah, G. PIN3 RELATIVE VACCINE EFFECTIVENESS OF QUADRIVALENT CELL-BASED VERSUS EGG-BASED INFLUENZA VACCINES AMONG ADULTS 50-64 YEARS OLD: A U.S. OBSERVATIONAL COHORT STUDY. Value Heal. 2020, 23, S168, https://doi.org/10.1016/j.jval.2020.04.479.

31. DeMarcus, L.; Shoubaki, L.; Federinko, S. Comparing influenza vaccine effectiveness between cell-derived and egg-derived vaccines, 2017-2018 influenza season. Vaccine 2019, 37, 4015-4021, https://doi.org/10.1016/j.vaccine.2019.06.004.

32. Izurieta, H.S.; Chillarige, Y.; Kelman, J.; Wei, Y.; Lu, Y.; Xu, W.; Lu, M.; Pratt, D.; Chu, S.; Wernecke, M.; et al. Relative Effectiveness of Cell-Cultured and Egg-Based Influenza Vaccines Among Elderly Persons in the United States, 2017-2018. J. Infect. Dis. 2018, 220, 1255-1264, https://doi.org/10.1093/infdis/jiy716.

33. Izurieta, H.S.; Chillarige, Y.; Kelman, J.; Wei, Y.; Lu, Y.; Xu, W.; Lu, M.; Pratt, D.; Wernecke, M.; MaCurdy, T.; et al. Relative Effectiveness of Influenza Vaccines Among the United States Elderly, 2018-2019. J. Infect. Dis. 2020, 222, 278-287, https://doi.org/10.1093/infdis/jiaa080.

34. Klein, N.P.; Fireman, B.; Goddard, K.; Zerbo, O.; Asher, J.; Zhou, J.; King, J.; Lewis, N. Vaccine effectiveness of cell-culture relative to egg-based inactivated influenza vaccine during the 2017-18 influenza season. PLOS ONE 2020, 15, e0229279, https://doi.org/10.1371/journal.pone.0229279.

35. Boikos, C.; Sylvester, G.C.; Sampalis, J.S.; A Mansi, J. Relative Effectiveness of the Cell-Cultured Quadrivalent Influenza Vaccine Compared to Standard, Egg-derived Quadrivalent Influenza Vaccines in Preventing Influenza-like Illness in 2017-2018. Clin. Infect. Dis. 2020, 71, e665-e671, https://doi.org/10.1093/cid/ciaa371.

36. Liberati, A.; Altman, D.G.; Tetzlaff, J.; Mulrow, C.D.; Gøtzsche, P.C.; Ioannidis, J.P.A.; Clarke, M.; Devereaux, P.J.; Kleijnen, J.; Moher, D. The PRISMA Statement for Reporting Systematic Reviews and Meta-Analyses of Studies That Evaluate Health Care Interventions: Explanation and Elaboration. PLoS Med. 2009, 6, e1000100, doi:10.1371/journal.pmed.1000100.

37. Moher, D.; Shamseer, L.; Clarke, M.; Ghersi, D.; Liberati, A.; Petticrew, M.; Shekelle, P.; Stewart, L.A. Preferred reporting items for systematic review and meta-analysis protocols (PRISMA-P) 2015 statement. Syst. Rev. 2015, 4, 1, https://doi.org/10.1186/20464053-4-1.

38. Moher, D.; Liberati, A.; Tetzlaff, J.; Altman, D.G.; The PRISMA Group. Preferred reporting items for systematic reviews and meta-analyses: The PRISMA Statement. PLoS Med. 2009, 6, e1000097-6, https://doi.org/10.1371/journal.pmed.1000097.

39. Ouzzani, M.; Hammady, H.; Fedorowicz, Z.; Elmagarmid, A. Rayyan-A web and mobile app for systematic reviews. Syst. Rev. 2016, 5, 210, doi:10.1186/s13643-016-0384-4.

40. Sterne, J.A.C.; Hernán, M.A.; Reeves, B.C.; Savović, J.; Berkman, N.D.; Viswanathan, M.; Henry, D.; Altman, D.G.; Ansari, M.T.; Boutron, I.; et al. ROBINS-I: a tool for assessing risk of bias in non-randomised studies of interventions. BMJ 2016, 355 , i4919. https://doi.org/10.1136/bmj.i4919.

41. Hernán MA, Robins JM. Causal Inference: What If. Online. Boca Raton: Champman \& Hall/CRC: Available online: https://cdn1.sph.harvard.edu/wp-content/uploads/sites/1268/2019/10/ci_hernanrobins_1oct19.pdf (accessed on day month year).

42. Halloran, M.E.; Longini, I.M.; Struchiner, C.J. Design and Analysis of Vaccine Studies. 2010, https://doi.org/10.1007/978-0-38768636-3.

43. Berman, N.G.; A Parker, R. Meta-analysis: Neither quick nor easy. BMC Med Res. Methodol. 2002, 2, https://doi.org/10.1186/14712288-2-10.

44. Higgins, J.P.T.; Thompson, S.G.; Deeks, J.J.; Altman, D.G. Measuring inconsistency in meta-analyses. BMJ 2003, 327, 557-560, doi:10.1136/bmj.327.7414.557.

45. Sterne, J.A.; Gavaghan, D.; Egger, M. Publication and related bias in meta-analysis: Power of statistical tests and prevalence in the literature. J. Clin. Epidemiol. 2000, 53, 1119-1129, doi:10.1016/s0895-4356(00)00242-0.

46. Duval, S.; Tweedie, R. Trim and Fill: A Simple Funnel-Plot-Based Method of Testing and Adjusting for Publication Bias in MetaAnalysis. Biometrics 2000, 56, 455-463, doi:10.1111/j.0006-341x.2000.00455.x.

47. Raudenbush, S.W. Analyzing effect sizes: Random-effects models. In The Handbook of Research Synthesis and Meta-Analysis, 2nd ed.; Russell Sage Foundation: New York, NY, USA, 2009; pp. 295-316.

48. Boikos,C.; Fischer, L.; O’Brien, D.; Vasey, J.; Gregg, C. Sylvester, J.A.M. Relative Effectiveness of Cell-Derived Quadrivalent Inactivated Influenza Vaccine (ccIIV4) Versus Egg-Derived IIV4 in Preventing Influenza-Related Medical En-counters During the 2018-2019 Influenza Season in the United States. In ESWI2020 Virtual Edition; Valencia, 6-9 December, 2020, p. Abstract 246.

49. Postma, M.; Pelton, S.I.; Divino, V.; Mould-Quevedo, J.F.; Anupindi, V.R.; Mitchell DeKoven, G.K. Relative vaccine effectiveness against influenza-related hospitalizations and any respiratory event during the 2018/19 high influenza activity period. RealWorld Analysis to compare quadrivalent cell-based and egg-based influenza vaccines. In ESWI2020 Virtual Edition; Valencia, 69 December, 2020, p. Abstract 214.

50. Boikos, C.; Imran, M.; Nguyen, V.H.; Ducret, T.; Sylvester, G.C.; Mansi, J.A. Relative Effectiveness of Cell-Derived versus Eggderived Quadrivalent Influenza Vaccines in Individuals with Underlying Medical Conditions in the U.S. 2018-2019 Influenza Season. In ESWI2020 Virtual Edition; Valencia, 6-9 December, 2020, p. Abstract 468. 
51. Pelton, S.I.; Postma, M.; Divino, V.; Mould-Quevedo, J.F.; Anupindi, V.R.; Mitchell DeKoven, G.K. Relative vaccine effectiveness against influenza-related hospitalizations and serious respiratory events during the 2018/19 influenza season in children and adults. Comparison between quadrivalent cell-based and egg-based influenza vaccin. In ESWI2020 Virtual Edition; Valencia, 69 December, 2020, p. Abstrcat 211.

52. Tseng, H.F.; Bruxvoort, K.J.; Luo, Y.; Anderson, B.; Tanenbaum, H.C.; Sy, L.S. Vaccine effectiveness against influenza hospitalization in the 2018-2019 season: comparison between cell-based and egg-based influenza vaccines. In Proceedings of the Options $X$ for the Control of Influenza, ISIRV, Singapore, 28 August-1 September, 2019. Abstract no: 10935

53. Krishnarajah, G.; Divino, V.; Postma, M.J.; Pelton, S.I.; Anupindi, V.R.; DeKoven, M.; Mould-Quevedo, J. Clinical and Economic Outcomes Associated with Cell-Based Quadrivalent Influenza Vaccine vs. Standard-Dose Egg-Based Quadrivalent Influenza Vaccines during the 2018-19 Influenza Season in the United States. Vaccines 2021, 9, 80, https://doi.org/10.3390/vaccines9020080.

54. Eick-Cost, A.A; Hu, Z. Relative Effectiveness of Cell-Based Influenza Vaccines Compared with Egg-based Influienza Vaccines, Active Component, U.S. Service Members, 2017-18 Season. In Proceedings of the International Conference on Emerging Infectious Diseases (ICEID2018), Atlanta, GA, USA, 26-29, August, 2018, p. 109.

55. Martin, E.T.; Cheng, C.; Petrie, J.G.; Alyanak, E.; Gaglani, M.; Middleton, D.B.; Ghamande, S.; Silveira, F.P.; Murthy, K.; Zimmerman, R.K.; et al. Low Influenza Vaccine Effectiveness Against A(H3N2)-Associated Hospitalizations in 2016-2017 and 2017-2018 of the Hospitalized Adult Influenza Vaccine Effectiveness Network (HAIVEN). J. Infect. Dis. 2020, https://doi.org/10.1093/infdis/jiaa685.

56. Izurieta, H.S.; Lu, M.; Kelman, J.; Lu, Y.; Lindaas, A.; Loc, J.; Pratt, D.; Wei, Y.; Chillarige, Y.; Wernecke, M.; et al. Comparative Effectiveness of Influenza Vaccines Among US Medicare Beneficiaries Ages 65 Years and Older During the 2019-2020 Season. Clin. Infect. Dis. 2020, https://doi.org/10.1093/cid/ciaa1727.

57. Boikos, C.; Fischer, L.; O’Brien, D.; Vasey, J.; Sylvester, G.C.; A Mansi, J. Relative Effectiveness of the Cell-derived Inactivated Quadrivalent Influenza Vaccine Versus Egg-derived Inactivated Quadrivalent Influenza Vaccines in Preventing Influenza-related Medical Encounters During the 2018-2019 Influenza Season in the United States. Clin. Infect. Dis. 2021, 73, e692-e698, https://doi.org/10.1093/cid/ciaa1944.

58. Okoli, G.N.; Racovitan, F.; Righolt, C.H.; Mahmud, S.M. Variations in Seasonal Influenza Vaccine Effectiveness due to Study Characteristics: A Systematic Review and Meta-analysis of Test-Negative Design Studies. Open Forum Infect. Dis. 2020, 7, ofaa177, https://doi.org/10.1093/ofid/ofaa177.

59. Skowronski, D.M.; Sabaiduc, S.; Leir, S.; Rose, C.; Zou, M.; Murti, M.; A Dickinson, J.; Olsha, R.; Gubbay, J.B.; Croxen, M.; et al. Paradoxical clade- and age-specific vaccine effectiveness during the 2018/19 influenza A(H3N2) epidemic in Canada: potential imprint-regulated effect of vaccine (I-REV). Eurosurveillance 2019, 24, 1900585, https://doi.org/10.2807/15607917.es.2019.24.46.1900585.

60. Ferdinands, J.M.; Gaglani, M.; Ghamande, S.; Martin, E.T.; Middleton, D.; Monto, A.S.; Silveira, F.; Talbot, H.K.; Zimmerman, R.; Smith, E.R.; et al. Vaccine Effectiveness Against Influenza-Associated Hospitalizations Among Adults, 2018-2019, US Hospitalized Adult Influenza Vaccine Effectiveness Network. J. Infect. Dis. 2020, 224, 151-163, https://doi.org/10.1093/infdis/jiaa772.

61. Paules, C.I.; Sullivan, S.G.; Subbarao, K.; Fauci, A.S. Chasing Seasonal Influenza - The Need for a Universal Influenza Vaccine. New Engl. J. Med. 2018, 378, 7-9, https://doi.org/10.1056/nejmp1714916.

62. Garten, R.; Blanton, L.; Elal, A.I.A.; Alabi, N.; Barnes, J.; Biggerstaff, M.; Brammer, L.; Budd, A.P.; Burns, E.; Cummings, C.N.; et al. Update: Influenza Activity in the United States During the 2017-18 Season and Composition of the 2018-19 Influenza Vaccine. MMWR. Morb. Mortal. Wkly. Rep. 2018, 67, 634-642, https://doi.org/10.15585/mmwr.mm6722a4.

63. Flannery, B.; Kondor, R.J.G.; Chung, J.R.; Gaglani, M.; Reis, M.; Zimmerman, R.K.; Nowalk, M.P.; Jackson, M.L.; A Jackson, L.; Monto, A.S.; et al. Spread of Antigenically Drifted Influenza A(H3N2) Viruses and Vaccine Effectiveness in the United States During the 2018-2019 Season. J. Infect. Dis. 2019, 221, 8-15, https://doi.org/10.1093/infdis/jiz543.

64. Xu, X.; Blanton, L.; Elal, A.I.A.; Alabi, N.; Barnes, J.; Biggerstaff, M.; Brammer, L.; Budd, A.P.; Burns, E.; Cummings, C.N.; et al. Update: Influenza Activity in the United States During the 2018-19 Season and Composition of the 2019-20 Influenza Vaccine. Morb. Mortal. Wkly. Rep. 2019, 68, 544-551, https://doi.org/10.15585/mmwr.mm6824a3.

65. Egger, M.; Smith, G.D.; Schneider, M.; Minder, C. Bias in meta-analysis detected by a simple, graphical test. BMJ 1997, 315, 629634, doi:10.1136/bmj.315.7109.629.

66. Orenstein, E.; De Serres, G.; Haber, M.J.; Shay, D.; Bridges, C.B.; Gargiullo, P.; A Orenstein, W. Methodologic issues regarding the use of three observational study designs to assess influenza vaccine effectiveness. Int. J. Epidemiol. 2007, 36, 623-631, https://doi.org/10.1093/ije/dym021.

67. Hamilton, M.A.; Calzavara, A.; Emerson, S.D.; Djebli, M.; Sundaram, M.E.; Chan, A.K.; Kustra, R.; Baral, S.D.; Mishra, S.; Kwong, J.C. Validating International Classification of Disease 10th Revision algorithms for identifying influenza and respiratory syncytial virus hospitalizations. PLOS ONE 2021, 16, e0244746, https://doi.org/10.1371/journal.pone.0244746.

68. Higgins, J.P.T.; Thompson, S.G. Quantifying heterogeneity in a meta-analysis. Stat. Med. 2002, 21, 1539-1558, https://doi.org/10.1002/sim.1186.

69. Sullivan, S.G.; Cowling, B. “Crude Vaccine Effectiveness” Is a Misleading Term in Test-negative Studies of Influenza Vaccine Effectiveness. Epidemiology 2015, 26, e60-e60, https://doi.org/10.1097/ede.0000000000000343. 